\title{
Creación musical en la era postdigital ${ }^{1}$
}

\section{Musical Creation in the Post-digital Era}

\author{
Rodrigo F. Cádiz \\ Pontificia Universidad Católica de Chile, Chile \\ rcadiz@uc.cl
}

- Resumen En el presente artículo se abordan algunos de los desafíos y características más relevantes de la creación musical en la era postdigital, desde el particular punto de vista de un compositor. Se otorga un especial énfasis a tres áreas al interior de la creación musical: la música electroacústica, la composición algorítmica y las nuevas interfaces de expresión musical. El artículo presenta tanto discusiones teóricas como ejemplos tomados de la producción artística de quien escribe.

Palabras clave: creación artística, era postdigital, música electroacústica, composición algorítmica, nuevas interfaces de expresión musical.

- Abstract - In this article, some of the most important features and challenges of musical creation in the post-digital era are discussed, from the particular viewpoint of a composer. Three areas of musical creation are given special consideration: electroacoustic music, algorithmic composition and new interfaces for musical expression. This article presents both theoretical discussions and examples taken from the author's artistic production.

Keywords: artistic creation, post-digital era, electroacoustic music, algorithmic composition, new interfaces for musical expression.

$1 \quad$ Este artículo forma parte del proyecto de investigación Fondecyt de Iniciación $\mathrm{N}^{\circ} 11090193$ y del proyecto del Fondo de Fomento de la Música Nacional Folio N 15872-0, Consejo Nacional de la Cultura y las Artes, Gobierno de Chile. 


\section{INTRODUCCIÓN}

Hace diez años, Weill (2002) hizo una crónica sobre el décimo aniversario del Salón Digital de Nueva York, el cual, según él, proveía una oportunidad única de observar el extraordinario cambio experimentado por la producción artística y cultural de la década anterior (1992 a 2002). Weill describe que en 1992 muy pocos artistas incorporaban en sus trabajos tecnología digital, y estos pertenecían a una minoría muy marginal cuya producción parecía estar muy determinada por la tecnología. En ese entonces, esta minoría pertenecía, en su mayoría, a la academia o a la esfera corporativa. La tecnología era cara y escasa y la pericia técnica necesaria para su manipulación era difícil de adquirir. En 2002, ya en plena era postdigital², Weill describe que en solo diez años la tecnología había permeado toda la cultura, se producía masivamente y se había vuelto cada vez más accesible, incluso para los artistas, quienes ahora producían trabajos que revelaban el extraordinario nuevo espectro de posibilidades de creación, sobre todo con la intervención del computador personal.

Actualmente, a diez años de la crónica de Weill, la influencia de la tecnología en todos los ámbitos de la creación y producción artística no ha hecho más que incrementarse. En 2009, Cadoz argumentaba que la tecnología constituía en muchos casos una condición para la existencia social, histórica y cultural de objetos y procesos de creación artística. Más aún, incluso el contenido de los objetos de arte puede ser dependiente de las condiciones tecnológicas que hacen posible su creación. Las interacciones entre la tecnología y el objeto artístico, el proceso de su creación, su conservación, difusión y existencia social son actualmente múltiples y complejas, pero, a su vez, cada vez más comunes.

El advenimiento de la tecnología digital, en contraposición a su antecesora análoga, no constituyó simplemente algo pasajero, ni solamente un cambio repentino en la evolución del desempeño o la precisión de ciertos sistemas (Cadoz 2009). Se trata de un cambio bastante más profundo, el cual no cabe del todo en la palabra digital $^{3}$. La industria digital es una de las pocas que ha experimentado un incremento dramático en funcionalidad al mismo tiempo que una igualmente drástica reducción en costos, gracias al cumplimiento prácticamente ininterrumpido por cuarenta años de la Ley de Moore, la cual predice un crecimiento exponencial del números de elementos que pueden ser contenidos por un circuito digital (Loy 23).

2 En 1998, Nicholas Negroponte, fundador del MIT Media Lab, publicó un artículo en la revista de tecnología Wired argumentando que la revolución digital había llegado a su fin, dando inicio a la era postdigital. Algunos autores, en particular Cascone (2000) y Turner (2003), asumen esta fecha como el inicio de lo que llaman música postdigital.

3 La palabra digital viene del término dígito, o número. La tecnología digital se basa en el concepto de número, su tratamiento y su cálculo (Cadoz 2009). Una de las características más importantes de la tecnología digital es que la información es discretizada para su procesamiento, esto es, se guardan solo ciertos valores numéricos de una señal continua tomados a intervalos regulares de tiempo. 
El término arte digital usualmente se utiliza para denominar expresiones artísticas dependientes y derivadas del uso de técnicas de computación digital binarias ${ }^{4}$, donde todo, incluyendo colores, sonidos, formas y movimiento son reducidos a un código con solo dos posibles estados (Rand 2008). Estos son solo dos debido a que los circuitos eléctricos que solo utilizan dos estados son mucho más estables que los de múltiples estados. Esta estabilidad permite que los elementos básicos de los circuitos digitales, llamados transistores, puedan ser muy pequeños y no pongan en riesgo la estabilidad de todo el sistema (Loy 22). En consecuencia, el computador solo reconoce estas dos posibles manifestaciones, y todo el arte digital emerge de esta capacidad, por cierto limitada, de los computadores de reconocer, almacenar, procesar y desplegar combinaciones de estas dos posibles situaciones.

Candy (2007) afirma que en las artes digitales el proceso creativo es el mismo que en otros campos creativos, el cual implica necesariamente establecer restricciones. Algunas de estas son elegidas por el artista, mientras que otras dependen del contexto, del género particular o del medio. En el caso del arte digital, la tecnología impone algunas restricciones adicionales que son inherentes a la naturaleza del computador, el cual es un medio relativamente inmaduro. Esto implica que han surgido un gran número de diferentes aproximaciones a la creación artística basada en tecnología, desde la digitalización de medios ya existentes a la generación de nuevas formas de arte, en las cuales la distinción entre el medio y la herramienta en muchos casos ya no existe. Rand (2008) sugiere que el arte digital debe tener como objetivo crear un mundo nuevo, una constante condición de sí... entonces, contraria a lo ya establecido, y no ser solamente una extensión trivial de los medios musicales o visuales, lo que él denomina arte asistido por computador.

Una de los aspectos más trascendentes para la creación artística que el uso de la tecnología permite es la revelación de principios estructurales profundos en las obras de arte. El computador, y en particular el uso de ciertos paradigmas de programación, provee un incremento significativo en la habilidad de manejar y considerar las estructuras subyacentes en obras y sistemas de producción de arte en cualquiera de las variadas formas que estos pueden tomar (Edmonds 2002). Candy (2007) profundiza en esta idea, mencionando que una característica muy importante de la tecnología digital es que, para ser utilizada en todo su potencial, es necesario estar preparado para explicitar cualquier presunción implícita en la mente del creador. Es esta imperiosa necesidad de explicitación la que hace el trabajo creativo desafiante y gratificante para muchos artistas.

Para trabajar digitalmente, las restricciones creativas deben ser explicitadas en forma tal que el computador genere un resultado satisfactorio para el creador. Sin embargo, Candy (2007) enfatiza que más importante que aquello es que el proceso

$4 \quad$ El término binario se refiere a un sistema de dos estados. Esto en principio no tiene nada que ver con el concepto digital. Un sistema digital podría ser no binario, pero, en el caso de los computadores de uso común basados en tecnología eléctrica, se utiliza un sistema de este tipo. Otros modelos teóricos de computación, por ejemplo, la computación cuántica, se basan en sistemas de múltiples estados. 
de la explicitación de las restricciones en un formato digital solo pueden ser entendido como una parte integral del proceso creativo. La atracción de lo digital para los creadores está muchas veces en relación con la capacidad del computador de representar y ejecutar la estructura subyacente en las obras de arte.

En el caso particular de la música, la influencia tecnológica es especialmente evidente. En 1966, a propósito del rol de la música en la era electrónica, el notable pianista Glenn Gould escribió que, gracias a la tecnología, especialmente las nuevas técnicas de grabación disponibles en esa fecha, las veneradas distinciones de la estructura musical que usualmente separan a compositor, intérprete y auditor tenderían a desaparecer (Gould 124), algo que hoy es evidente en ciertos géneros o prácticas musicales, como por ejemplo los mash-up (Moorefield 108). Pocos años antes, Edgar Varèse, uno de los compositores más influyentes del siglo xx , en 1962 aseguraba que este nuevo medio tecnológico le había otorgado a los compositores posibilidades casi ilimitadas de expresión, y les había abierto de par en par las puertas del misterioso mundo del sonido (Varèse 20).

La introducción del computador como herramienta en la creación musical ha generado profundas consecuencias para la evolución del medio tecnológico como un todo (Manning, Electronic and computer music 181). Una de las principales razones que llevan a un compositor a componer música mediante computadores es poder pensar y crear música en forma distinta a como sería sin un computador (Taube 5). En este sentido, Ferreira (2001) afirma que la exploración del universo sonoro, que se hace posible mediante la tecnología electrónica y computacional, implica necesariamente una reformulación de lo que constituye la problemática musical. Esta exploración ya no es simplemente un problema musical puro enmarcado en un pensamiento que posiciona las actividades artísticas y científicas en polos opuestos. Al contrario, se hace posible exactamente por los avances tecnológicos, implicando incluso que la música tenga que compartir con la tecnología parte de sus propios problemas. Ferreira cita el ejemplo de que problemas en las áreas de modelamiento matemático y el procesamiento de datos, ya constituyen territorio común para las artes.

Considerando otros aspectos de la experiencia musical, el desarrollo de recursos para la grabación y reproducción de sonidos ha tenido consecuencias de largo alcance para la práctica de la música. Usualmente el proceso de la síntesis y la comunicación requería de la presencia física de un intérprete y audiencia en una experiencia común. Hoy la intimidad social de una performance en vivo en la sala de conciertos es reemplazada por una emisión comercial o un disco compacto en los cuales los procesos intermedios de grabación y edición tienen un impacto significativo en lo que le es entregado a los auditores. Esta intervención de la tecnología ha creado un continuo interesante entre la búsqueda de la autenticidad de la reproducción y la tentación de experimentar con el medio como nuevas formas de expresión musical (Manning 2003). El ambiente digital actual de la creación, producción y la experiencia musical no separa claramente los roles tradicionales de la composición como 
un acto privado de ver y la escucha como un acto público de examinación, sino que más bien los mezcla y confunde (Everett 2001).

El destacado compositor chileno Gabriel Brnčić (2011), sintetiza muy bien las ideas expuestas hasta ahora:

las técnicas de síntesis electrónica de formas de ondas, las que a través de altavoces prefiguran un nuevo repertorio instrumental, junto a las técnicas de grabación de sonido -equivalentes a la fotografía y a la grabación cinematográfica- además de la amplificación de cualquier suceso acústico, para su difusión masiva, han ensanchado el horizonte auditivo en el siglo xx y han comprometido a todo un espacio de actividad social-industrial basado en la 'cultura del altavoz' (40).

En la misma línea de argumentación, Rowe (2001) escribe que la tecnología computacional no solamente ha hecho posible nuevas formas de creación, sino que también ha provocado un gran revuelo en las prácticas musicales artísticas y culturales a todo nivel. La tecnología hoy es barata, fácil de usar y no se cansa.

Considerando que, en la actualidad, tecnología y arte son inseparables en muchos casos, es pertinente y urgente reflexionar sobre los cambios artísticos que se producen debido a la dramática y acelerada transformación que la tecnología ha experimentado en el último tiempo. Crear música hoy, en esta era postdigital, claramente no es igual que hace veinte años, en pleno desarrollo de la era digital. Este artículo pretende revisar algunos aspectos relevantes en la creación digital de música, mediante una revisión teórica y la ilustración de ciertas problemáticas abordadas en la obra artística reciente de quien escribe.

A continuación se discute y ejemplifica el uso de diversas herramientas tecnológicas y digitales en tres niveles distintos de la creación musical. En primer lugar, se aborda el uso de la tecnología en la generación y manipulación directa de los sonidos, lo cual es la esencia del género denominado música electroacústica. En segundo término se abarca el campo del diseño de estrategias para la combinación de sonidos en el tiempo, mediante herramientas computacionales, el cual se conoce como composición algorítmica. Finalmente, se revisa la aplicación y creación de tecnología con el fin de generar nuevas posibilidades de expresión en la producción e interpretación de material sonoro, bajo el título nuevas interfaces de expresión musical.

\section{MÚSICA ELECTROACÚSTICA}

La música electroacústica, entendida como aquella que es generada mediante aparatos electrónicos o mediante una combinación de estos con instrumentos acústicos (Cádiz 2003), constituye un género bastante particular en la historia de la música y manifiesta grandes diferencias con respecto a la música instrumental (Pope 1994). Tanto es así que algunos compositores de música electroacústica han llegado a cuestionar si lo que hacen puede ser considerado música, en el sentido en que normalmente 
entendemos el término. Otros prefieren la denominación «arte sonoro» y muchos incluso rechazan la idea de ser compositores en el sentido tradicional. Por lo general, comprenden a la música como un subconjunto de este amplio arte auditivo (Morthenson 1985) y según Jean-Claude Risset, este nuevo arte se constituye de hecho en otra rama de la música, tan distinta de la música instrumental como lo es el cine del teatro (Risset 2002). Otros autores como Delalande plantean que el arte musical ha experimentado tres momentos históricos: la tradición oral, la escrita (mediante una partitura) y la electroacústica, mediante la fijación en un medio (Landy 178).

La notación musical tradicional, al ocuparse fundamentalmente de las alturas, duraciones, e intensidades de los sonidos, no permite transmitir todo el rango de información detallada del fenómeno sonoro que sí es captado por el sistema auditivo humano. Varèse, en 1936, proponía que una nueva notación musical, de tipo sismográfica, sería mucho más apropiada que la tradicional (Varèse 18). La aparición de la tecnología de la grabación sonora cambió radicalmente esta situación, permitiendo fijar en forma permanente una señal acústica con todos sus detalles. Cadoz (2009) menciona que, en forma paralela, la electricidad y la electrónica pusieron en circulación un objeto novedoso: la señal. De esta manera, los procesos de transmisión, transformación, procesamiento y generación de señales se convirtieron en nuevas formas de creación y diseminación de material sonoro.

Esto dio lugar a la llamada música concreta en 1948, que tuvo sus orígenes en Francia, mientras que la música electrónica surgió en Alemania en 1950. Ambas propuestas constituyen las raíces de lo que hoy se conoce como música electroacústica, y las dos cambiaron radicalmente el concepto de composición. En el caso de la música concreta, propuesta por Pierre Schaeffer, el proceso de creación es novedoso ya que, una vez que los eventos sonoros se fijan en un medio permanente, se convierten en lo que él llama objetos sonoros (Schaeffer). Mediante el término música concreta Schaeffer quiso enfatizar que esta nueva música provenía de material sonoro concreto, escuchado en forma explícita con la intención de abstraer valor musical de aquel. Esto resulta opuesto a la música clásica, la cual parte de una concepción abstracta que se convierte, mediante mediación de intérpretes, en una performance concreta (Landy 77).

El caso de la música electrónica es levemente distinto, ya que no persigue solamente componer con sonidos, sino que componer los sonidos (Stockhausen 88-9). Hasta 1950, escribe Stockhausen, la idea de la música como sonido era largamente ignorada. El hecho de que componer música también podría involucrar componer los sonidos no era algo evidente, y se constituyó en la principal bandera de este género.

Solo diez años después de la aparición de la música concreta y la música electrónica, el computador hace su triunfante aparición en el dominio musical mediante lo que se denominó composición algorítmica (Hiller e Isaacson 1959) y la síntesis digital de sonidos (Mathews 1963), dando paso luego a la llamada música computacional (Roads 1999). Los computadores hoy están presentes en todas las fases del trabajo de un compositor, desde exploraciones precomposicionales hasta 
la producción sonora, pasando también por la notación y fijación del sonido en un soporte físico (Taube 4).

Hoy existen numerosas manifestaciones y vertientes distintas de la música electroacústica, la cual, tal como propone Delalande (cit. en Landy 178), quizás ya no debiera llamarse de esta forma, sino música tecnológica. Algunas denominaciones originales ya no se usan, como por ejemplo música electrónica, término que fue apropiado por tendencias de carácter más popular y menos experimentales. La música acusmática, denominación también propuesta por Schaeffer y derivada de la música concreta, se utiliza actualmente para denominar una experiencia particular de escucha donde las fuentes sonoras no son reveladas. Otras vertientes, como la música interactiva (Winkler 1998), música de laptop (Turner 2003), glitch (Cascone 2000), circuit bending (Ghazala 2004), o live coding (Collins et al. 2003) han ido expandiendo los horizontes de la música electroacústica hacia áreas hasta hace poco tiempo insospechadas.

No obstante esta riqueza de variantes, en la música electroacústica suele suceder que el acto compositivo y el control paramétrico de los algoritmos de procesamiento y síntesis llevados a cabo en el computador están absolutamente entrelazados y son imposibles de separar (Cádiz 2006, Chadabe 1997; Dahlstedt 2001; Manning 2004; Wolek 2005). En estas situaciones, la composición se convierte y confunde con el acto de controlar adecuadamente los parámetros del proceso computacional. Como consecuencia, el proceso compositivo se ve fuertemente moldeado por la naturaleza de la síntesis o procesamiento empleados. La composición mediante computadores requiere que el compositor tenga un entendimiento profundo de las técnicas de síntesis y procesamiento que utiliza (Burt 1996; Castagne y Cadoz 2002; Wolek 2005). A medida que nuevas formas de procesamiento y síntesis son propuestas, el modo de componer cada obra en particular se ve afectado (Bernardini y Rudi 2002).

La síntesis digital de sonidos es un área dentro de la música que ha emergido a una velocidad sin precedentes. Hace cincuenta años, los primeros sonidos producidos digitalmente otorgaron a los compositores de todo el mundo una pequeña muestra de inmensas nuevas posibilidades artísticas. Cadoz (2009) explica que, al digitalizarse el sonido, se debe obtener una cantidad muy grande de números por cada segundo del fenómeno acústico con el objetivo de satisfacer la gran cantidad de información que el sistema auditivo humano requiere para una correcta audición ${ }^{5}$. Mediante la síntesis de sonidos es posible establecer vínculos entre algoritmos complejos de manipulación y organización de estos grandes conjuntos de datos y nuestra percepción.

En consecuencia, experimentos numéricos con el sonido se han convertido en una extensión lógica de intereses composicionales que exploraban las fronteras del

En concreto, se requieren exactamente 44.100 muestras por segundo para digitalizar un sonido en calidad de CD. Este valor se obtiene mediante el Teorema del Muestreo, que dice que es necesario digitalizar una señal con una frecuencia mayor que el doble de la frecuencia más alta de la señal a ser digitalizada. En el caso de la audición humana, esta frecuencia es de 20.000 ciclos por segundo, aproximadamente. 
timbre, percepción, nuevos instrumentos y técnicas de interpretación, entre otros. Alentados por las posibilidades, muchos centros de investigación formaron equipos interdisciplinarios de científicos, ingenieros y músicos para combinar composición con investigación en acústica musical, psicoacústica e inteligencia artificial (Chafe 1999). Como resultado, numerosas técnicas de síntesis digital de audio, basadas en distintos principios, han sido diseñadas e implementadas, muchas de ellas comercialmente.

La mayoría de las técnicas tradicionales de síntesis, por ejemplo, las basadas en la serie de Fourier ${ }^{6}$, o en modulación ${ }^{7}$, tienen como objetivo el producir sonidos relativamente simples mediante modelos sencillos que no requieran de una gran capacidad de procesamiento computacional y que puedan funcionar en tiempo real (Roads 1999). Esto se debe fundamentalmente a que la capacidad de los computadores era muy limitada en un comienzo, y generar unos pocos segundos de sonido digital requería usualmente de varias horas de procesamiento computacional. Las técnicas tradicionales de síntesis permiten generar en forma bastante fidedigna sonidos de tipo instrumental o natural, muchos de los cuales están basados en la serie armónica $^{8}$, son altamente redundantes en el sentido de que sus distintas bandas de frecuencia contienen la misma información desde el punto de vista estadístico (Attias y Schreiner 1997), y tienen una huella característica, dado que concentran su energía en las zonas bajas de modulación temporal y espectral (Singh y Theunissen 2003).

En general, sonidos más complejos (musicalmente más atractivos) como los llamado ecológicos (Keller, 2000; Oliveira y Oliveira 2002), denominación basada en el trabajo perceptual de Gibson (1966), presentan dificultades para su modelamiento sonoro porque sus características temporales y espectrales están altamente correlacionadas, y por lo tanto, no pueden ser generados mediante técnicas tradicionales de síntesis (Keller y Berger 2001). En forma adicional, poco se sabe sobre la identificación y percepción de este tipo de sonidos (Ballas 1993). Esto explica por qué hay muchas técnicas de síntesis que permiten emular sonidos instrumentales, pero hay muy pocas que tratan de emular sonidos más complejos.

La generación de sonidos complejos puede ser abordada mediante otro tipo de técnicas, como por ejemplo, la síntesis granular (Roads 2001), la cual se basa en

6 Las series de Fourier constituyen la herramienta matemática básica del análisis de Fourier empleado para analizar funciones periódicas a través de la descomposición de dicha función en una suma infinita de funciones sinusoidales mucho más simples. El nombre se debe al matemático francés Jean-Baptiste Joseph Fourier, quien desarrolló esta teoría en 1807.

7 Existen dos variantes principales de la síntesis por modulación: la basada en modulación de amplitud (AM) y de frecuencia (FM), respectivamente. En la síntesis AM, la amplitud de un sonido es variado de acuerdo a otro sonido y, en la síntesis FM, es la frecuencia o fase la que es variada de acuerdo a otro sonido de referencia.

8 La serie armónica es una sucesión de sonidos cuyas frecuencias son múltiplos enteros positivos de la frecuencia de una nota base, llamada fundamental. Estas frecuencias corresponden exactamente a las de la serie de Fourier sobre esa nota fundamental. En una teoría simplificada del timbre musical, cada uno de los sonidos de la serie armónica es un componente del timbre o color del sonido representado por una nota cuya frecuencia es la del sonido fundamental. A los sonidos de la serie armónica, componentes del timbre, se les llama armónicos. 
la idea de pequeños granos de sonidos $^{9}$ que son combinados para formar sonidos más complejos o el modelamiento físico (Smith, 1992), que busca modelar la fuente generadora del sonido y no el sonido en sí. Otros autores han propuesto métodos basados en ideas tan diversas como la teoría de caos, el modelamiento dinámico y atractores sonoros (Röbel 2001), transformaciones paramétricas sobre dimensiones perceptualmente relevantes (Keller y Berger 2001), percepción de eventos (Gaver 1993), modelamiento espectral (Amatriain et al. 2002), mecánica cuántica (Ramos y Cádiz 2010), física de partículas y la Ley de De Broglie (Sturm 2001), resonancia estocástica (Cádiz y de la Cuadra 2008 y 2010) o la síntesis basada en sistemas complejos (Huepe et al. 2012).

\section{EJEMPLOS}

En parte de mi creación artística, la composición de la obra y las técnicas de síntesis y procesamiento de sonidos utilizadas se entrecruzan de manera fundamental. A continuación se detallan algunos aspectos relevantes de obras que hacen patente esta situación.

El género de música para instrumentos solistas es algo propio de la música contemporánea (con la excepción de instrumentos solistas por naturaleza como el piano o el violín), dado que en los repertorios barroco, clásico y romántico, usualmente estos instrumentos han sido utilizados en el contexto de una agrupación instrumental, ya sea de cámara u orquestal. Existen muchos compositores contemporáneos que han exhibido un marcado interés en explorar la veta solística a través de la creación de series de piezas solistas, en donde cada pieza está dedicada a explorar las posibilidades sonoras de un instrumento en particular. Como ejemplos notables se pueden citar las Sequenzas de Luciano Berio, los Memos de Bernard Rands, las obras solistas de Brian Ferneyhough y Salvatore Sciarrino, y, en Chile, los Solitarios de Alejandro Guarello. Todos estos compositores buscan estudiar el fenómeno del instrumento solo, totalmente expuesto, desprotegido, desnudo, sin la presencia de otras fuentes sonoras de las cuales nutrirse y en las cuales apoyarse. Este tipo de composición obliga al compositor a llevar la técnica instrumental al límite, de manera de crear sonoridades novedosas. En este sentido, la música solista contemporánea ha dado lugar a nuevas técnicas de ejecución que de otra manera no hubiesen emergido.

Parte de mi creación musical toma como base este trabajo instrumental anterior, pero buscando nuevos contextos sonoros. Mi propuesta contempla la utilización de sonidos instrumentales en un contexto electroacústico, donde los sonidos son modificados computacionalmente y expuestos en secuencias musicales más complejas, las cuales no podrían ser realizadas mediante la ejecución de instrumentos acústicos por seres humanos. En el fondo de mi propuesta subyace la misma idea anterior de

En la síntesis granular, cada grano de sonido, análogo a una partícula de luz, tiene una duración entre cinco y cincuenta milisegundos, dependiendo de su contenido de frecuencia. 
la exploración del material sonoro de un instrumento determinado, pero el interés real es explorar las posibilidades del sonido instrumental, no las del instrumento ni del ejecutante. El foco de mi trabajo es el sonido, no el instrumento físico.

La mayoría de los compositores de música electroacústica basan su trabajo en la utilización de sonidos concretos proveniente de distintas fuentes, por lo general tomadas del medio ambiente, o sonidos artificiales creados mediante distintos algoritmos computacionales, o en la interacción de sonidos generados acústicamente por instrumentos reales con paisajes sonoros de música electroacústica. Pero, solo en contadas ocasiones, el compositor hace uso de sonidos que provengan solamente de una misma fuente y de un instrumento acústico determinado. Tampoco es común el caso de compositores que exploren sistemáticamente sonidos de instrumentos musicales procesados mediante tecnología digital. Una excepción importante de mencionar es el trabajo electroacústico de Pierre Boulez, en piezas como Dialogue de l'Ombre Double o Antheme II, en las cuales el sonido del instrumento solista, clarinete y violín en este caso, interactúa con sonidos provenientes del mismo instrumento y levemente procesados computacionalmente. Pero a Boulez, tomando en cuenta la totalidad de su producción artística, la música concreta o la música puramente electrónica no le son de gran interés, y estas obras son solo dos trabajos puntuales y no constituyen una exploración sistemática. Otro caso a mencionar es el de Mario Davidovsky, mediante sus Synchronisms para distintos instrumentos y grupos de cámara y medios electroacústicos. En este caso, a pesar de que hay una intención de explorar la interacción de instrumentos acústicos con medios electrónicos, no se trata siempre de instrumentos solistas, y, en muchos casos, los sonidos electrónicos no son derivados de los instrumentos. En otras palabras, los Berio, Rands, Ferneyhough, Sciarrino o Guarello en la música electroacústica son escasos. Al parecer, la necesidad de explorar las sonoridades de distintos instrumentos acústicos en contextos electroacústicos solistas no es algo común.

Esta necesidad se manifiesta no solo desde el punto de vista musical, sino más bien desde una perspectiva más global, al observar cómo ciertos métodos, objetos y creencias muy arraigadas en nuestras culturas en el pasado han sido modificadas por la tecnología computacional. Un ejemplo claro de esto es el eMail, forma de comunicación que ha reemplazado al tradicional correo (mail en inglés) o el eCommerce, nueva forma de hacer comercio por Internet. En el ámbito académico, el estudio de la cultura digital ha tenido un auge importante en las últimas décadas. Un ejemplo a citar es el trabajo realizado en el Media Lab del Massachusetts Institute of Technology (MIT) sobre eRationality, ${ }^{10}$ definida como el estudio de las formas en que la gente se comporta y toma decisiones en el día a día, particularmente en ambientes electrónicos. Diversas revistas y conferencias se dedican anualmente al estudio de estos tópicos.

$\overline{10}$ Mayor información sobre este concepto, se puede encontrar en http://erationality.media.mit.edu/. 
Musicalmente, esta preocupación se evidencia en mi obra $e P i a n o^{11}$, para soporte fijo ${ }^{12}$ estéreo, compuesta el año 2003 mientras realizaba mis estudios de doctorado en Northwestern University. Esta obra nace a partir de unas pocas muestras de piano como material inicial, las que son transformadas mediante diversas técnicas como remuestreo, procesos de filtrado y síntesis granular, de manera de proponer nuevas dimensiones auditivas del mundo sonoro del piano. El auditor siempre tendrá como referencia el mundo acústico tradicional del piano, pero experimentará el material en formas nuevas e impredecibles. De esta forma, el marco de referencia sonoro tradicional y conocido del piano se ve ampliado y transformado, alcanzando sonoridades insospechadas.

ePiano pertenece a una serie de obras electroacústicas solistas rotuladas con el texto eInstrument, tomando como patrón los conceptos descritos anteriormente. Otras obras que han sido compuestas bajo este paradigma hasta la fecha son $e Q$ uena ${ }^{13}$, para quena y electrónica en vivo ${ }^{14}$, eRecorder ${ }^{15}$, para flauta dulce contrabaja y electrónica en vivo y, finalmente, $e$ Chant $^{16}$, para cantante tenor y electrónica en vivo. Los nombres de las obras están a propósito en inglés, para reflejar la influencia de la cultura anglosajona, principal generadora de la tecnología que actualmente nos domina.

11 ePiano fue seleccionada y estrenada en el Festival June in Buffalo, en la Universidad de Buffalo en Buffalo, Nueva York, en junio de 2004. También fue presentada en el IV Festival Internacional de Música Electroacústica de Santiago, Ai-maako, en octubre de 2004, en el Octavo Electronic Music Midwest Festival en Kansas City, EEUU, en octubre de 2006, y en Silencio 08, en Santa Fe, Argentina, en mayo de 2008. ePiano fue incluida en el CD «50 años de música electroacústica en Chile» y en el disco «UniSono», disco doble que contiene obras acústicas y electroacústicas compuestas por quien escribe.

12 El término "soporte fijo» se utiliza cuando la música se presenta en concierto a partir de una grabación, usualmente en formato digital. Antes de la aparición de los CDs o discos duros portátiles, comúnmente se utilizaba el término "cinta».

13 eQuena fue estrenada por Patricio de la Cuadra en octubre de 2008 en el VIII Festival Internacional de Música Electroacústica de Santiago Ai-maako, en el Centro Cultural de España, y se encuentra editada en el disco «UniSono».

14 Usualmente el término «electrónica en vivo» se utiliza cuando el audio de un instrumento musical es procesado mediante técnicas electrónicas en tiempo real, a diferencia del término "soporte fijo» o electrónica a secas, que usualmente implican la utilización de pistas de material electrónico previamente grabadas en superposición con el sonido en vivo del instrumento.

15 eRecorder fue estrenada por Paola Muñoz en noviembre de 2009 en el XIX Festival de Música Contemporánea Chilena, en el Salón Fresno del Centro de Extensión de la Pontificia Universidad Católica de Chile. Posteriormente fue interpretada en 2010 por Terri Hron en Santiago y Valdivia, en el X Festival Aimaako y en Nueva York en la International Computer Music Conference. eRecorder se encuentra editada en el disco «UniSono".

16 eChant fue estrenada por José Quilapi en octubre de 2009 en el Concierto GEMA en la Sala Isidora Zegers de la Universidad de Chile y posteriormente presentada en el IX Festival Aimaako, en el Centro Cultural Matucana 100, y se encuentra editada en el disco «UniSono». 
Sintética ${ }^{17}$ es una obra que utiliza solamente sonidos generados mediante síntesis $^{18}$ en tiempo real, sin utilizar sonidos grabados de ninguna especie, en forma intencional. En Sintética, la composición y la creación del sonido se funden a tal punto que ya no son separables. Esto contrasta claramente con otras obras, donde predomina el material grabado o bien hay una mezcla entre ambos. De esta forma, Sintética es un intento creativo por validar la generación de sonido de forma algorítmica como una herramienta de un gran potencial artístico. En este sentido, me interesa explorar la síntesis como medio de generación de material sonoro, pero también como articulación del discurso musical.

El material sonoro de Sintética guarda una relación directa y muy fuerte con la síntesis utilizada en la composición, dado que la mayoría de las técnicas de síntesis presentes en esta obra provienen de la familia de la síntesis espectral, que consiste en algoritmos y procedimientos realizados directamente en el dominio de la frecuencia y no en el dominio temporal. Este tipo de procedimientos permite de cierta manera moldear el contenido espectral de un sonido con un gran nivel de detalle. A través de estas técnicas es también posible mezclar propiedades de dos sonidos distintos y generar sonidos híbridos que, por ejemplo, tienen una estructura de frecuencias proveniente de una fuente sonora, pero con un comportamiento temporal que proviene de otra distinta. La utilización de este tipo de sonidos es algo característico de Sintética, y que define gran parte de su propuesta artística.

Otra característica importante de esta obra es su naturaleza de tiempo real, dado que el material sonoro de la obra no se encuentra fijado en un soporte como es el caso, por ejemplo, de ePiano, sino que se genera en tiempo real mediante una ejecución o interpretación de la obra en el escenario, lo que la emparenta con otras obras abordadas en la sección relacionada con las nuevas interfaces de expresión musical.

Imber $^{19}$, compuesta en 2011 en las dependencias del Centro de Investigación en Tecnologías de Audio y el Laboratorio de Tecnología Musical de la Pontificia Universidad Católica de Chile, es una obra que explora muchas de las posibilidades del agua como medio de expresión musical. La palabra Imber proviene del latín y se podría traducir como lluvia o precipitación. En Imber, todos los sonidos se originan en la interacción entre agua y distintos materiales. En la composición se utilizaron sonidos de lluvia, gotas de agua resonando en distintos ambientes, tormentas, agua pasando por todo tipo de cañerías, agua en la ducha, en el lavamanos, en el baño,

17 Sintética fue estrenada en octubre de 2009 en el concierto Ciclo Electro-LAIM, en la Sala SCD, y fue posteriormente presentada en el en el IX Festival de Música Electroacústica de Santiago Ai-maako, en el Centro Cultural Matucana 100. Ambas presentaciones fueron interpretadas en vivo por quien escribe, mediante un sistema de sensores gestuales programados especialmente para esta obra.

18 La síntesis de sonidos busca crear y generar sonidos mediante algoritmos numéricos o procedimientos computacionales, sin recurrir a sonidos grabados o tomados del mundo natural. En contraste, el procesamiento de sonidos toma como fuente sonidos grabados y los modifica mediante diversas técnicas y procedimientos.

19 Imber fue estrenada en octubre de 2011 en el XI Festival Ai-maako, realizado en el Centro Cultural Gabriela Mistral. También fue seleccionada para su estreno en Europa en la International Computer Music Conference, en Ljubljana, Eslovenia, en septiembre de 2012. 
agua en ebullición, vapor de agua y sonidos del mar. La mayoría de estos sonidos fueron modificados mediante diversas técnicas de procesamiento de audio en el computador. En este sentido, el material sonoro de Imber contrasta claramente con el de Sintética, al tratarse de sonidos grabados y procesados, sin ningún origen sintético. Sintética e Imber se encuentran en extremos opuestos desde el punto de vista de las estrategias de composición utilizadas, una basada en síntesis versus otra fundada en procesamiento.

Dado que la mayoría de los sonidos utilizados en Imber comparten un origen común, el trabajo de composición se basa en la adecuada combinación de manifestaciones sonoras distintas de un mismo material original. Desde este punto de vista, se trata de una obra que explora en mayor medida el timbre como la propiedad organizadora de los contrastes sonoros. Este es un aspecto de la composición en que la tecnología digital disponible actualmente es de mucha utilidad, dado que mediante una gran variedad de herramientas de análisis y procesamiento de audio es posible analizar con gran precisión muchas de las características del timbre de un sonido, con el objetivo de tomar decisiones de composición que sin estas herramientas sería muy difícil realizar. La tecnología permite estructurar música en base a dimensiones sonoras que usualmente no son utilizadas en la creación musical, como es en este caso el timbre, el cual no constituye comúnmente la base de la organización del material musical en la música, salvo escasas excepciones (Patel 30-7).

La tecnología también es clave en otro aspecto de la composición de Imber: la generación de material rítmico a partir de sonidos que no presentan mayor información rítmica en su versión original. Mediante ciertas técnicas de procesamiento computacional es posible utilizar ciertas secciones de los sonidos para generar repeticiones, variaciones y texturas rítmicas de gran complejidad, las cuales son utilizadas en ciertos lugares estratégicos de la composición.

Por último, es necesario destacar la naturaleza multicanal ${ }^{20}$ de esta obra, la cual fue compuesta especialmente para un sistema de reproducción de ocho canales de audio, algo que es bastante común en el género de la música electroacústica. Un computador permite organizar la información en un número casi ilimitado de canales de audio, lo cual facilita enormemente la generación de música especialmente pensada para funcionar en muchos planos y dimensiones distintas. Si bien en los inicios de la música electroacústica muchas obras multicanales fueron compuestas con equipamiento electrónico análogo, la tecnología digital sin lugar a dudas facilita y hace posible la creación de música que no sigue el formato estéreo, el cual aún domina en la grabación y difusión de gran parte de la música que se crea en la actualidad.

20 En el género de la música electroacústica es frecuente presentar música en más de dos parlantes a la vez. Usualmente se utilizan cuatro, ocho, dieciséis o más canales de audio independientes, distribuidos espacialmente en la sala de conciertos de manera de generar una imagen espacial sonora determinada. La espacialidad es un factor muy importante en la composición de música electroacústica. 


\section{COMPOSICIÓN ALGORÍTMICA}

El elevado grado de abstracción y formalización que posee la música, en sus aspectos teóricos, ha llevado a menudo a compararla con las matemáticas. No sin razón, a una de las más importantes disciplinas musicales, la armonía, suele elevársele a la categoría de ciencia. Se ha afirmado, por ejemplo, que los músicos crearon los sistemas de coordenadas antes que los estudiosos de los números, ya que una partitura musical es realmente un sistema de coordenadas donde son representados valores de frecuencia en el eje de las ordenadas, y el transitar del tiempo en el eje de las abscisas (Hinojosa 2005).

Dada la complejidad inherente a la composición musical, surge, en la década de los cincuenta, el área de la composición musical asistida por computador. En la actualidad, las computadoras suelen ser empleadas para diferentes fines dentro de la composición de música. En primer lugar, funcionando como un secuenciador, permite la verificación inmediata de lo que se está componiendo, lo cual es particularmente útil cuando se busca lograr efectos tímbricos nuevos y por lo tanto desconocidos. En segundo lugar, puede colaborar en la realización de cálculos tediosos, especialmente combinatorios, requeridos para organizar una forma musical compleja. En tercer lugar, puede componer automáticamente en base a una serie de pautas introducidas para tal efecto. Por último, tal como se ha expuesto en forma anterior, permite trabajar íntimamente con el sonido mismo, mediante síntesis y análisis, en donde la elaboración de un sonido complejo, de un timbre nuevo, reviste igual importancia que su aplicación posterior a la composición de una obra.

Diversos tipos de algoritmos (o sistemas de reglas formales) se han utilizado para componer música por siglos; como un ejemplo se pueden citar los procedimientos de conducción de voces en el contrapunto occidental, los cuales pueden ser modelados algorítmicamente en forma bastante certera. El término, sin embargo, generalmente es reservado para el uso de procedimientos formales de composición musical sin la intervención humana. No obstante, en el contexto de este artículo, el término composición algorítmica se entiende en su concepción más amplia, es decir, como la aplicación de algoritmos a la composición musical en cualquiera de sus etapas creativas y con intervención humana.

Un algoritmo puede ser entendido de tres maneras (Nierhaus 2). En primer lugar, como un conjunto de instrucciones matemáticas que deben ser seguidas en un orden particular. En segundo, como un procedimiento sistemático que produce la solución a un problema en un número finito de pasos. Finalmente, como usualmente se entiende en el campo de la inteligencia artificial, un algoritmo se define como un conjunto de reglas o procesos finitos y predeterminados, con una o más etapas, que tiene como objetivo encontrar uno o más resultados a un problema determinado. En un sentido amplio, se trata de operaciones lógicas de carácter sintáctico que pueden transformar un input en un output deseado. Los algoritmos 
pueden ser aplicados de manera eficiente a innumerables áreas, como el área matemática o, en nuestro caso, la música.

Los algoritmos no son un producto de la tecnología digital, y ya existían en la música mucho antes de la invención del computador serial digital, como por ejemplo en la creación de Guido d'Arezzo en 1025, Bach (Nierhaus 2010) o Mozart (Manzolli 1996, 2000, cit. en Oliveria y Zampronha 2002). Otro caso interesante es el del músico teórico ruso, emigrado a Estados Unidos, Joseph Schillinger, quien, en la década de los años veinte y treinta, desarrolló un detallado sistema de composición musical basado en principios científicos. Este sistema está compuesto por siete libros, cada uno de ellos centrado en un aspecto diferente de la composición musical. Según Pérez (2000), la obra de Schillinger todavía no goza del reconocimiento que según algunos merece, pero ha influido enormemente en la música del siglo xx , especialmente en compositores como George Gershwin, Glenn Miller o Benny Goodman, entre otros. La base del sistema de Schillinger es geométrica y se fundamenta en el concepto de relaciones de fase de movimientos periódicos simples. Schillinger encontró distintas formas de proyectar estas relaciones en el ritmo, pero también en áreas mucho menos obvias como el tono, la escala, los acordes, la progresión armónica e, incluso, en los aspectos semánticos y emocionales de la composición musical. Hay quienes consideran que el sistema de Schillinger anticipó la música computacional antes de que existieran los computadores, y que introdujo muchas técnicas algorítmicas de composición, incluso la utilización de series numéricas autosemejantes (Pérez 2000).

Un algoritmo, en general, implica una serie de condicionantes. Los algoritmos, en muchos casos, constituyen prohibiciones (o restricciones). Los elementos de la música con los cuales trabaja el compositor, así como las combinaciones posibles de estos elementos, constituyen un conjunto finito enormemente grande de valores. La función del compositor es seleccionar un subconjunto de valores de este gran conjunto, darles una determinada organización temporal que cumpla con ciertas reglas musicales, y crear una obra de arte. Claros ejemplos al respecto son el Arte de la Fuga de Bach o la música dodecafónica de Schoenberg (Nierhaus, 2010).

De acuerdo con Oliveira y Zampronha (2002), el sistema tonal, que se consolidó a partir del barroco y continuó hasta el final del siglo xIx, con reflejos importantes hasta hoy, es lo que se puede llamar un sistema musical consolidado. No es por azar que gran parte de las investigaciones que relacionan la composición musical con la ciencia cognitiva concebida dentro del cognitivismo clásico, principalmente dentro de la inteligencia artificial, trabajen sobre este sistema. Ser un sistema musical consolidado significa tener reglas codificadas y bien definidas, las cuales son aplicadas a materiales musicales conocidos y también codificados.

Estas condiciones son importantes para que un sistema composicional pueda ser reproducido por algoritmos. Así, los discursos musicales resultantes pueden ser comparados con paradigmas formales y estéticos también consolidados, de modo que la eficiencia del algoritmo puede ser evaluada. Sin embargo, es más difícil con- 
cebir un algoritmo para producir obras que pertenezcan a sistemas musicales no consolidados. Principalmente, aquellos que no operan con una materia sonora que pueda ser fácilmente transformada en representaciones simbólicas, y cuyas reglas sintácticas son difíciles de ser formalizadas. La música concreta, por ejemplo, adopta como punto de partida la selección de los materiales por la escucha. No hay aquí representaciones simbólicas tan explícitas.

Desde un punto de vista computacional, la composición algorítmica se relaciona directamente con áreas de la inteligencia artificial ${ }^{21}$ como son la programación de restricciones (Hinojosa 2005), autómatas celulares (Nierhaus 2010), redes neuronales (Nierhaus 2010) y los algoritmos basados sistemas complejos (Huepe et al. 2012). La creciente capacidad computacional disponible para los músicos ha hecho posible trabajar a través del control indirecto de unidades que ya no precisan de una instrucción precisa del compositor para cada una de las acciones a realizar. Así, un generador de ideas (el compositor, en nuestro caso) podía instruir a un tercero (la computadora) para que aplicara las indicaciones y reglas de bajo nivel que éste le indicara. Esto libera al compositor de la toma de decisiones para cada detalle, permitiéndole ocuparse de un control de alto nivel y enfocarse sobre los gestos musicales, en caso que así lo deseara (Dal Farra 2011).

Desde la segunda mitad del siglo xx , las disciplinas de la música y matemáticas, arte y ciencia, comienzan a reencontrarse gracias al uso extendido de los computadores y programas de procesamiento digital de señales. La primera pieza musical completamente generada por un computador en un nivel simbólico ${ }^{22}$ fue producida por Hiller e Isaacson en 1956 en la universidad de Illinois y llamada Illiac Suite (Nierhaus 2010; Taube 2004). A medida que los diversos lenguajes computacionales conocidos hoy se fueron generando, también se desarrollaron sofisticados sistemas de composición algorítmica. Algunos de ellos se basan en la extensión de conceptos de los lenguajes tradicionales de propósito general y otros en paradigmas puramente musicales.

La estructura musical generada a partir de algoritmos puede provenir desde prácticamente cualquier disciplina científica (Nierhaus 2010). Se necesita generar lo que se denomina un mapeo, es decir una relación formal entre elementos de alguna estructura o paradigma particular y elementos musicales. Se pueden utilizar algoritmos para tratar solo un aspecto particular de una pieza musical o para determinar la forma global de esta (Nierhaus 2010). Ambas alternativas implican el uso de enfoques bastante específicos y posiciones estéticas diferenciadas.

Según Taube (2004), en el campo de la composición algorítmica pueden distinguirse al menos tres variantes dependiendo de la forma de utilización y aplicación del computador a la composición. En primer lugar, se puede hablar de composición

21 La inteligencia artificial se podría definir como la combinación de diversas disciplinas que tratan con la automatización de un comportamiento inteligente (Nierhaus, 2010: 225).

22 Un nivel simbólico en este caso implica que el computador genera como salida una representación de valores musicales que deben ser interpretados por un músico. 
asistida por el computador, donde este facilita tareas como el cálculo de datos precomposicionales, la edición de eventos o la reproducción sonora de material. En general, en esta situación, el computador se utiliza antes o después de la generación de una idea musical en la mente del compositor, y no como una forma de representación de formalismos composicionales. En segundo término, se puede considerar la composición automática, realizada por sistemas computacionales para componer música en forma independiente al ser humano. Uno de los ejemplos más notables en este campo es el trabajo de David Cope (1996, 2000), quien desarrolló sistemas computacionales que permiten a un computador componer música en estilo de una alta verosimilitud y musicalidad. Por último, Taube describe la composición basada en computadores, un meta-nivel en el cual estos son utilizados en forma explícita para representar ideas composicionales a un nivel superior que la mera notación o partitura. En este meta-nivel, las relaciones y los procesos que constituyen una composición son representadas al interior de la máquina.

\section{EJEMPLOS}

En varias de mis composiciones he utilizado técnicas de composición algorítmica, en cada una de las variantes descritas por Taube. Tres de los ejemplos más recientes y relevantes en este sentido son mis obras Tensiones, de 2008, y Extensiones y Divergencias, ambas compuestas el año 2010.

Extensiones $^{23}$ es un ejemplo de composición asistida por computador. Se trata de una obra para percusión solista, orquesta y electrónica en tiempo real y fue compuesta especialmente para el percusionista Gerardo Salazar y la Orquesta Sinfónica de Chile. El material sonoro de la obra se deriva de un principio composicional denominado serie infinita ${ }^{24}$, propuesto originalmente por el compositor danés Per Nørgård a fines de los años cincuenta (Anderson 1997). La serie infinita consiste en una secuencia de números enteros que puede formarse mediante la sucesiva réplica e inversión de un intervalo musical. Esta serie posee propiedades únicas de autosimilitud, repeticiones y simetrías, y puede programarse en forma bastante simple en un computador actual.

En Extensiones, el material de la serie infinita se va presentando de distintas formas y toma distintas manifestaciones al transcurrir la música, tanto en forma acústica como electrónica. En particular, el material musical inicial del vibráfono al comienzo de la pieza consiste en una expansión de la serie infinita centrada en la nota central del registro del instrumento. Fragmentos de esta serie se van tras-

23 Extensiones fue estrenada por Gerardo Salazar y la Orquesta Sinfónica de Chile, bajo la dirección de Michal Nesterowicz, en octubre de 2010. La composición de esta obra fue posible gracias al apoyo del Fondo de Fomento de la Música Nacional y al Consejo Nacional de la Cultura y las Artes, del Gobierno de Chile.

24 Esta secuencia se encuentra registrada en la enciclopedia online de series de números enteros (OEIS) y puede ser consultada en la dirección http://oeis.org/A004718. 
pasando gradualmente hacia los otros instrumentos de la orquesta a medida que el tiempo transcurre.

En Extensiones, el rol de la tecnología digital no constituye un mero acompañamiento al material sinfónico, sino que interactúa con este a varios niveles, modificando directamente el resultado sonoro producido por la orquesta. Esta misma búsqueda fue realizada anteriormente en la obra Tensiones ${ }^{25}$, para percusión virtual, ensamble y electrónica en vivo, compuesta en 2008 para el mismo Gerardo Salazar y el Taller de Música Contemporánea UC. Extensiones extiende algunas de las ideas y aproximaciones contenidas en Tensiones, de ahí deriva su título. Tensiones, a diferencia de Extensiones, es un ejemplo de composición basada en computadores, donde la tecnología computacional se utiliza en prácticamente todo el proceso, desde la generación de material precomposicional hasta la generación cuasi automática de la partitura. Tensiones se aborda en mayor detalle en la sección de nuevas interfaces de expresión musical.

Divergencias $^{26}$ es una obra para cuatro instrumentos de teclado de percusión: glockenspiel, xilófono, vibráfono y marimba. El material utilizado en esta obra también se basa en la serie infinita, pero utilizada de una forma distinta a como se utiliza en Extensiones, tomando en cuenta la particular naturaleza de la instrumentación elegida para la obra. A partir de una secuencia única, presentada al inicio en los cuatro instrumentos, se van produciendo variaciones y una gradual separación del material de alturas en los diversos instrumentos, hasta llegar a un punto máximo de separación e independencia de las voces. En forma adicional se utilizaron patrones rítmicos basados en la serie y en ciertos métodos estocásticos, como por ejemplo el movimiento browniano ${ }^{27}$. El juego entre la coincidencia total y el desfase de los patrones rítmicos en las cuatro voces es una de las características más importantes de esta obra. El proceso de composición de Divergencias se aproxima en cierta medida a la composición automática, dado que a través de un programa computacional, donde se especifican ciertas reglas de generación y restricción del material musical, se genera la partitura directamente desde el software, donde el compositor especifica las condiciones iniciales y las reglas de evolución temporal de la música, pero el computador se encarga de realizar todos los cálculos y poner las notas musicales en los lugares correctos para su posterior interpretación por el cuarteto de percusión.

25 Tensiones fue estrenada en noviembre de 2008 en el marco del XVIII Festival de Música Contemporánea Chilena, en el Salón Fresno del Centro de Extensión de la Pontificia Universidad Católica de Chile, por el Taller de Música Contemporánea UC, bajo la dirección de Pablo Aranda. Tensiones se encuentra editada en el disco Panorama Electroacústico 2010, producido por el Laboratorio Arcis de Informática Musical con el apoyo del Consejo Nacional de la Cultura y las Artes.

26 Divergencias fue estrenada en noviembre de 2010 en el Xx Festival de Música Contemporánea Chilena, en la Sala 1 del Centro Cultural Gabriela Mistral, por el Ensamble de Percusión UC, bajo la dirección de Gerardo Salazar.

27 El movimiento browniano es un tipo de movimiento aleatorio que se observa naturalmente en algunas partículas microscópicas que se hallan en un medio fluido. Un ejemplo de este tipo de movimiento es el que experimenta el polen en una gota de agua. 


\section{NUEVAS INTERFACES DE EXPRESIÓN MUSICAL}

Un instrumento musical puede entenderse como una interfaz que intermedia gestualidad y sonido (Roads 1999). Un instrumento permite a un intérprete manipular y controlar el sonido de acuerdo a ciertas acciones o gestos musicales. En la tradición de la interpretación musical, la relación entre el músico y su instrumento se desarrolla a un nivel muy alto. Largos años de práctica diaria son necesarios para adquirir un conocimiento profundo y cabal del instrumento en todos sus atributos físicos y mecánicos antes de que el músico pueda decir que se ha dominado su instrumento. Este proceso de aprendizaje implica una relación dinámica que va mucho más allá del simple dominio de la técnica, en el sentido de que pone en juego las cualidades musicales de la interacción personal que se ha desarrollado entre el músico y su instrumento. Desde esta perspectiva, la relación entre el músico y su instrumento es mucho más compleja de lo que podríamos esperar de la interacción típica entre un ser humano y una máquina.

Una razón obvia de esto es que nuestra relación con un instrumento musical se basa en el tiempo que pasamos viviendo con él (Tanaka 2000). En el desarrollo de instrumentos acústicos, los diseñadores tienen que encontrar un equilibrio óptimo entre las capacidades del cuerpo humano y las limitaciones físicas que intervienen en la producción de sonido. Los gestos utilizados en el acto de tocar un instrumento dependen en gran medida de las características física del instrumento (Arfib et al. 2005). Un músico que toca un instrumento musical tradicional debe suplir la energía para controlar y al mismo tiempo producir el sonido, mientras que y todos los instrumentos musicales acústicos están limitados por sus propiedades físicas y mecanismos. Por medio del instrumento, el artista es capaz de afectar todos los aspectos de la música, desde el nivel micro del timbre al nivel de la articulación de los eventos, hasta el nivel macro que refleja la estructura de la composición (Tanaka 2000). Es por lo tanto, imposible para un músico ir en contra de la propia naturaleza del instrumento, lo cual constituye un problema importante en la música de arte contemporánea que usualmente busca utilizar recursos sonoros fuera de los límites de los instrumentos acústicos. Los instrumentos musicales acústicos han evolucionado significativamente a través de los siglos hasta haberse estabilizado en formas canónicas, alcanzando un perfecto equilibrio entre todas las variables que determinan su usabilidad: ergonomía, flexibilidad interpretativa, potencial expresivo y diseño estético (Paradiso y O’Modhrain 2003).

En contraste, la tecnología electrónica provee un nuevo paradigma para el diseño de instrumentos musicales, mediante el uso de dispositivos de control novedosos que traducen el movimiento humano en señales eléctricas, las cuales son a su vez utilizadas para generar sonido en formas diversas. Esto permite expandir el horizonte de posibilidades de control y ejecución del material musical, una vieja aspiración de compositores como Varèse, quien, en 1939, soñaba con un medio de expresión musical totalmente nuevo, lo que él llamaba una máquina de producción 
sonora y no meramente una de reproducción sonora (Varèse 19), un dispositivo que permitiera tanto crear como controlar el sonido a voluntad.

De acuerdo a Roads (1999) el uso de dispositivos de control como parte de un instrumento musical tiene dos grandes ventajas por sobre los instrumentos tradicionales. En primer lugar, estos dispositivos de control electrónicos separan el control del sonido de su producción. Esto es algo que diferencia este nuevo paradigma de los instrumentos acústicos tradicionales en los cuales la producción y el control del sonido son inseparables. En segundo término, los instrumentos electrónicos pueden producir timbres diversos y pueden ser afinados o desafinados fácilmente. Esto otorga flexibilidad, dado que un dispositivo de control puede controlar varios generadores de sonido distintos, incluso en forma simultánea, y cambiar fácilmente propiedades como su contenido de frecuencia o afinación. Como las propiedades físicas del controlador no determinan ni condicionan las propiedades acústicas del sonido producido, esto permite que los intérpretes efectivamente sean independientes de cualquier ley física en términos de la producción sonora y a los compositores disponer de un universo sonoro ilimitado para crear música.

Los diseñadores de instrumentos de este tipo son libres de elegir la vinculación entre los gestos y los sonidos producidos (Arfib et al. 2005). Los instrumentos musicales electrónicos han hecho posible la disociación de la superficie de control (por ejemplo, las teclas, controles o válvulas) del dispositivo de generación de sonido (por ejemplo, los altavoces) (Mulder 2000). Durante muchos años, el teclado y sus perillas de control fueron los dispositivos de interfaz estándar utilizados en el género de la música electrónica. Hoy una gran cantidad de trabajo experimental que conecta teorías del campo denominado interfaces humano-computador (HCI) con la interpretación musical (O’Modhrain 2011). La gran variedad de sensores disponibles en la actualidad ha hecho posible la traducción de prácticamente cualquier gesto físico del mundo real en energía eléctrica, de modo que puedan utilizarse como señal de control y fuente de sonido electrónico (Bongers 2000). Crear una apropiada dinámica entre el artista y su instrumento es un objetivo importante en el diseño de instrumentos electrónicos, dado que implica la creación de las condiciones materiales que le permitirá al ejecutante alcanzar un nivel adecuado de dominio técnico y fluidez en la interpretación. El cómo lograr esta fluidez musical intuitiva con un instrumento basado en la tecnología constituye un desafío artístico de alta exigencia (Tanaka 2000).

Diversos tipos de instrumentos musicales electrónicos han estado disponibles por más de un siglo, durante el cual avances rápidos, muchas veces exponenciales, en la tecnología, han abierto continuamente nuevas posibilidades para la síntesis y el control, manteniendo este campo de investigación en una revolución continúa. Un gran número de instrumentos, controladores y ambientes musicales interactivos se han desarrollado a una gran velocidad y usualmente se ha adoptado el paradigma "controlador / computación / contenido", el cual es subyacente a la mayoría de los ambientes de programación musical disponibles de hoy (Machover 2002). 
Magnusson y Hurtado (2007) estudiaron la relación entre los intérpretes y sus instrumentos musicales a través de una encuesta abierta en Internet. La encuesta abordaba distintos problemas en el ámbito de los instrumentos acústicos y digitales, mediante preguntas sobre el control, la entropía instrumental, las limitaciones y la creatividad. El enfoque utilizado en la encuesta fue fenomenológico, es decir, se refiere a la experiencia de tocar, componer y diseñar instrumentos digitales y acústicos. Los resultados de la encuesta muestran contrastes importantes entre la percepción de los instrumentos acústicos y digitales, existiendo en este último caso una postura más crítica acerca de su diseño.

El diseño de un instrumento musical electrónico a menudo se superpone con la composición musical en sí, es decir, se diseñan instrumentos específicamente para una obra en particular y, por lo general, no son reutilizados en otras obras, dando lugar a una clara distinción en el mundo de los instrumentos acústicos que tienden a ser más generales con el fin de permitir la interpretación de una mayor variedad de piezas musicales. Esto podría explicar por qué la continuidad en el tiempo de los instrumentos digitales o interfaces de control a través del tiempo no es habitual.

Probablemente el instrumento de música electrónica más importante en la actualidad consiste en un controlador basado en sensores conectado a un computador genérico, el cual produce directamente y en tiempo real señales de audio altamente complejas. Dada la gran gama de sensores y la tecnología disponible en la actualidad, este controlador puede tomar las formas y principios de funcionamiento más diversos. Un instrumento de este tipo, dependiendo de la capacidad de computación disponible, permite el uso de un número arbitrario de parámetros de síntesis y algoritmos de control gestual altamente sofisticados. Cuando los sensores son usados para capturar gestualidad y un computador se ocupa para generar el sonido, un enorme rango de posibilidades se pone a nuestra disposición.

\section{EJEMPLOS}

El primero de los antecedentes más relevantes en esta área es mi obra Tensiones, compuesta en 2008 para solista virtual, ensamble y medios electrónicos. Esta obra tiene la particularidad que el solista no toca instrumentos acústicos tradicionales, sino que manipula directamente tanto los sonidos solistas como del ensamble a través de tecnología computacional en tiempo real. Si bien el solista es un percusionista, la pieza rompe con el rol del solista vs. acompañamiento. Aquí el solista modifica el resultado del acompañamiento, no solo «tocan juntos». Decide gran parte del resultado sonoro global, no solo de su parte particular. La pieza también explora nuevas formas de expresión musical, distintas a los instrumentos tradicionales. La obra fue escrita para el percusionista Gerardo Salazar, solista de la Orquesta Sinfónica de Chile, quien no opera directamente el computador, sino que utiliza dos controla- 
dores Wii Remote ${ }^{28}$ que se conectan inalámbricamente al computador y funcionan como instrumentos de percusión virtuales. El usar estos controladores permite, por ejemplo, cambiar la altura de los sonidos de los instrumentos de percusión, cosa que no se puede hacer físicamente.

Otros antecedentes son mis obras eQuena, eRecorder y eChant, todas para instrumentos solistas y electrónica en vivo, y mencionadas anteriormente en la sección sobre música electroacústica. Estas piezas forman parte de una serie de obras donde un instrumento acústico solista ve significantemente extendidas sus posibilidades sonoras a través de la tecnología digital, mediante la utilización de sensores e interfaces gestuales, permitiendo al intérprete extender las posibilidades sonoras del instrumento acústico a través de gestos que se traducen directamente en el procesamiento digital de la señal de audio proveniente del instrumento.

Otro ejemplo relevante es mi obra $i$ Cons $^{29}$ (2007), la cual es una obra interactiva multicanal para computador y sistema de sensores gestuales. La obra explora una sola fuente sonora, una moneda, la cual resuena de acuerdo a su interacción con distintos materiales y es procesada de diversas maneras en tiempo real. La exploración exhaustiva de las posibilidades musicales de una sola fuente sonora constituye un importante hilo conductor en mi obra, tal como se mencionó anteriormente. iCons también explora nuevas técnicas de expresión e interpretación musical ya que la evolución temporal y espacial de la obra se controla a través de un sistema de sensores en tiempo real desarrollado y manipulado por quien escribe. Este sistema permite controlar en forma gestual distintos aspectos del fenómeno sonoro que la pieza propone. El sistema gestual consiste en tres sensores de distancia basados en ultrasonido, además de una variedad de gatillos conectados al computador. Dicho sistema permite un enfoque bastante más musical al control del sonido que un teclado o un mouse. Mediante el solo movimiento de las manos es posible controlar la mayoría de los aspectos del sonido, tales como su forma en el tiempo y su posicionamiento espacial, o crear complejas densidades espectrales. En ciertos aspectos, este dispositivo funciona de manera similar a un Theremin ${ }^{30}$, con la diferencia que el sonido producido se encuentra totalmente separado del sistema de control. iCons es una pieza originalmente compuesta para cuatro canales, pero su diseño permite presentaciones a ocho canales o también estéreo. $i$ Cons fue concebida para

28 Wii es una consola de videojuegos fabricada por la compañía japonesa Nintendo. La característica más distintiva de la consola es su mando inalámbrico, el Wii Remote, el cual puede ser usado como un dispositivo de mano con el que se puede apuntar, además de detectar movimientos de los brazos y manos en un plano tridimensional.

29 iCons fue estrenada por quien escribe en octubre de 2007 en el VII Festival Internacional de Música Electroacústica de Santiago Aimaako, en el Centro Cultural de España. Posteriormente fue presentada en otros conciertos en Chile, Argentina y Portugal. $i$ Cons se encuentra editada en el disco «UniSono».

30 El Theremin, inventado por Leon Theremin en 1919, es uno de los primeros instrumentos musicales electrónicos. Funciona en base a campos electromagnéticos que son alterados mediante el movimiento de las manos, produciendo sonido. Una mano controla la frecuencia del sonido, mientras la otra manipula la amplitud. 
ser interpretada en vivo mediante el sistema de sensores descrito y su existencia es inseparable de la interfaz de expresión.

Un último ejemplo es la obra Extensiones, abordada anteriormente. A diferencia del formato tradicional para orquesta y electrónica donde esta se presenta en un soporte fijo, la electrónica, en esta obra, está «viva»; el computador reacciona en tiempo real a la ejecución del solista y de la orquesta y se convierte en un intérprete más del conjunto. El computador conoce y sigue la partitura al igual que cualquier músico de la orquesta, lo que le permite generar de manera instantánea respuestas y reacciones sonoras a lo que está sucediendo. Extensiones también explora, a través de la tecnología, la gestualidad del solista, ya que en forma adicional a la ejecución musical de los instrumentos acústicos, sensores especializados capturan información proveniente de los gestos del intérprete, la cual se utiliza en la generación y manipulación del material musical. En concreto, los instrumentos que el percusionista solista interpreta son un vibráfono, un triángulo, un iPad y un iPod ${ }^{31}$. Tanto el iPad como el iPod contienen sensores de aceleración en las tres dimensiones del espacio y giroscopios, lo que permite saber la posición angular de estos dispositivos en todo momento. El vibráfono y el triángulo son ejecutados de forma tradicional, mientras que el iPad es ejecutado en ciertas secciones a través de una interfaz gráfica especialmente diseñada y construida para la obra y mediante el movimiento del aparato en el espacio, el cual se encuentra especificado en la partitura y permite alterar la producción sonora del solista y la orquesta. El iPod se encuentra adosado al brazo del solista para capturar aceleración en los tres ejes del espacio y utilizar estos datos en el procesamiento del sonido producido por el solista y la orquesta. La utilización de estos dispositivos de uso común hoy como instrumentos musicales en la orquesta sinfónica resulta particularmente interesante. Extensiones, hasta donde el autor tiene entendido, es la primera obra a nivel mundial en utilizar un iPad como un instrumento sinfónico, lo cual confirma que la tecnología disponible ha permeado y seguirá permeando la creación y producción musical a todo nivel.

\section{REFERENCIAS}

Arfib, Daniel, Jean-Michel Couturier y Loïc Kessous. «Expressiveness and digital musical instrument design». Journal of New Music Research 34, 1 (2005). 125-36. Medio impreso.

Attias, Hagai y Christoph. E. Schreiner. «Temporal Low-Order Statistics of Natural Sounds». Advances in neural information processing systems 9 (1997). 27-33. Medio impreso.

31 El iPad y el iPod son dispositivos de tamaño pequeño fabricados por la empresa Apple, los cuales tienen funcionalidades muy similares a un computador genérico, además de contar con sensores especializados de movimiento. Más información se puede encontrar en http://www.apple.com. 
Amatriain, Xavier, Jordi Bonada, Alex Loscos y Xavier Serra. «Spectral Processing». DAFX: Digital Audio Effects. Ed. Udo Zoelzer. Chichester: John Wiley \& Sons, 2002. Medio impreso.

Anderson, Martin. «The many patterns of Per Nørgård». Tempo (New Series) 202 (1997). 3-7. Medio impreso.

Ballas, James A. "Common Factors in the Identification of an Assortment of Brief Everyday Sounds». Journal of experimental psychology: human perception and performance 19, 2 (1993). 250-67. Medio impreso.

Bernardini, Nicola y Joran Rudi. «Compositional use of digital audio effects». Journal of New Music Research 31, 2 (2002). 87-91. Medio impreso.

Bongers, Bert. «Towards a choice of gestural constraints for instrumental performers». Trends in Gestural Control of Music. Eds. Marcelo Wanderley y Marc Battier. París: IRCAM, 2000. Medio impreso.

Brnčić, Gabriel. «Sintéticos *Analíticos_Analíticos* Sintéticos (presente tenso)». Revista Musical Chilena 65, 215 (2011). 40-53. Medio impreso.

Burt, Warren. Some parenthesis around algorithmic composition. Organised Sound 1, 3 (1996). 167-72. Medio impreso.

Cádiz, Rodrigo. "Compositional Control of Computer Music by Fuzzy Logic». Tesis doctoral. Northwestern University, Evanston, Illinois, 2006.Tesis de Doctorado.

---. Estrategias auditivas, perceptuales y analíticas en la música electroacústica. Resonancias 13 (2003). 47-65. Medio impreso.

Cádiz, Rodrigo y Patricio De la Cuadra. «Spectral Stochastic Resonance Sound Synthesis». Proceedings of the International Computer Music Conference. Nueva York, 2010. Conferencia.

---. «Stochastic Resonance Sound Synthesis». Proceedings of the International Computer Music Conference. Belfast, 2008. Conferencia.

Cadoz, Claude. «Supra-Instrumental Interactions and Gestures». Journal of New Music Research 38, 3 (2009). 215-30. Medio impreso.

Candy, Linda. Constraints and creativity in the digital arts. Leonardo 40, 4 (2007). 366-7. Medio impreso.

Cascone, Kim. "The aesthetics of failure: 'post-digital' tendencies in contemporary computer music». Computer Music Journal 24, 4 (2000). 12-8. Medio impreso.

Castagne, Nicolas y Claude Cadoz. «Creating music by means of «physical thinking»: The musician oriented genesis environment». 5th international Conference on Digital Audio Effects (Dafx-02). Hamburgo, 2002. 169-74. Medio impreso.

Chadabe, Joel. Electric sound: the past and promise of electronic music. Upper Saddle River, New Jersey: Prentice Hall, 1997. Medio impreso.

Chafe, Chris. "A Short History of Digital Sound Synthesis by Composers in the USA». $<$ http://ccrma.stanford.edu/ cc/lyon/historyFinal.pdf>. Sitio web.

Collins, Nick, Alex Mclean, Julian Rohrhuber y Adrian Ward. «Live coding in laptop performance». Organised Sound 8 (2003). 321-30. Medio impreso. 
Cope, David. Experiments in Musical Intelligence. Madison: A-R Editions, 1996. Medio impreso.

---. The Algorithmic Composer. Madison: A-R Editions, 2000. Medio impreso.

Dal Farra, Ricardo. «Interacciones electroacústicas (entre la composición, la improvisación y la comprovisación)». En el límite 2, 2 (2011). 54-65. Medio impreso.

Dahlstedt, Palle. «Creating and exploring huge parameter spaces: Interactive evolution as a tool for sound generation». Proceedings of the International Computer Music Conference. La Habana, 2001. Conferencia.

Edmonds, Ernest A. «Structure in Art Practice: Technology as an Agent for Concept Development». Leonardo 35, 1 (2002). 65-71. Medio impreso.

Everett, Steve. Logics of Value for Digital Music. Proceedings of the International Conference Music Without Walls? Music Without Instruments? De Montfort University, 2001. Conferencia.

Ferreira, Giselle Martins Dos Santos. «When Art, Science and Technology meet: the Strength, the Dilemma or the Curse of Electroacoustic Music?». Proceedings of the International Conference: Music Without Walls? Music Without Instruments? De Montfort University, 2001. Conferencia.

Gaver, William. «Synthesizing auditory icons». Proceedings of INTERCHI '93 Amsterdam, 1993. 228-35. Conferencia.

Gibson, James J. The Senses Considered as Perceptual Systems. Boston: Houghton Mifflin, 1966. Medio impreso.

Gould, Glenn. «The Prospects of Recording». Audio Culture. Readings in Modern Music. Eds. Christoph Cox y Daniel Warner. New York: Continuum, 2010. Números de páginas del capítulo. Medio impreso.

Ghazala, Qubais Reed. «The Folk Music of Chance Electronics: Circuit-Bending the Modern Coconut». Leonardo Music Journal 14 (2004). 97-104. Medio impreso.

Hiller, Lejaren y Leonard Isaacson. Experimental Music: Composition with an Electronic Computer. New York: McGraw-Hill, 1959. Medio impreso.

Hinojosa, Rubén. «La composición algorítmica como satisfacción de restricciones». Fecha de ingreso: 19 de abril de 2012. <http://www.hinojosachapel.com/data/ texts/la_composicion_algoritmica_como_csp.pdf >. Sitio web.

Huepe, Cristian, Rodrigo Cádiz y Marco Colasso. «Generating Music from Flocking Dynamics». Proceedings of the American Control Conference, Montreal, 2012. Conferencia.

Keller, Damian. «Compositional Processes from an Ecological Perspective». Leonardo Music Journal 10, 1 (2000). 55-60. Medio impreso.

Keller, Damian y Jonathan Berger. «Everyday Sounds: Synthesis Parameters and Perceptual Correlates». VIII Brazilian Symposium on Computer Music. Brasil, 2001. Conferencia.

Landy, Leigh. Understanding the Art of Sound Organization. Cambridge: The MIT Press, 2007. Medio impreso. 
Loy, Gareth. Musimathics. The Mathematical Foundations of Music. Cambridge: The MIT Press, 2007. Medio impreso.

Magnusson, Thor y Enrike Hurtado. «The Acoustic, the Digital and the Body: A Survey on Musical Instruments». Proceedings of the 2002 Conference on New Interfaces for Musical Expression. Nueva York, 2007. Conferencia.

Machover, Tod. «Instruments, interactivity, and inevitability». Proceedings of the 2002 Conference on New Interfaces for Musical Expression. Dublin, 2002. Conferencia.

Manning, Peter. Electronic and computer music. New York: Oxford University Press, 2004. Medio impreso.

---. «The Influence of Recording Technologies on the Early Development of Electroacoustic Music». Leonardo Music Journal 13 (2003). 5-10. Medio impreso.

Mathews, Max. «The digital computer as a musical instrument». Science 142 (1963). 553-7. Medio impreso.

Moorefield, Virgil. The Producer as Composer. Shaping the Sounds of Popular Music. Cambridge: The MIT Press, 2005. Medio impreso.

Morthenson, Jay. "The concept of meaning in electronic music». Proceedings of the ICEM Conference on Electro-acoustic Music. Estocolmo, 1985. Conferencia.

Mulder, Alex. "Towards a choice of gestural constraints for instrumental performers». Trends in Gestural Control of Music. Eds. Marcelo Wanderley y Marc Battier. París: IRCAM, 2000. Medio impreso.

Negroponte, Nicholas. «Beyond Digital». Wired 6, 12 (1998). Medio impreso.

Nierhaus, Gerhard. Algorithmic Composition. Paradigms of Automated Music Generation. Wien: Springer-Verlag, 2010. Medio impreso.

Oliveira, Andre y Luis Oliveira. «Toward an ecological conception of timbre». Proceedings Auditory Perception Cognition and Action Meeting, Kansas City, 2002. Conferencia.

Oliveira, Luis y Edson Zampronha. «El computacionalismo clásico y el modelo de una mente creativa en composición musical». Actas de la segunda reunión de la Sociedad Argentina de las Ciencias Cognitivas de la Música. Argentina, 2002. Medio impreso.

O'modhrain, Sile. "A framework for the evaluation of digital musical instruments». Computer Music Journal 35, 1 (2011). 28-42. Medio impreso.

Patel, Aniruddh. Music, Language and the Brain. Oxford: Oxford University Press, 2008. Medio impreso.

Paradiso, Joseph A. y Sile O'modhrain. «Current trends in electronic music interfaces». Journal of New Music Research 32, 5 (2003). 345-9. Medio impreso.

Pérez, Juan Antonio. «Música fractal: el sonido del caos». (2000). Fecha de ingreso: 19 de abril de 2012. <http://www.dlsi.ua.es/ japerez/pub/pdf/mfsc2000.pdf>. Sitio web.

Pope, Steven T. «Why is good electroacoustic music so good? Why is bad electroacoustic music so bad?». Computer Music Journal 18, 3 (1994). 5-6. Medio impreso. 
Ramos, Javier y Rodrigo Cádiz. "Sound synthesis model based on the simulation of a Gaussian bouncing wave packet». Proceedings of the International Computer Music Conference, Huddersfield, Reino Unido, 2010. Conferencia.

Rand, Henry. «The other side of digital art». Leonardo 41, 5 (2008). 543-7. Medio impreso.

Risset, Jean Claude. «Prefacio». Electroacoustic Music. Analytical perspectives Ed. Thomas Licata. Westport: Greenwood Press, 2002. Medio impreso.

Roads, Curtis. Microsound. Cambridge: The MIT Press, 2001. Medio impreso.

---. The computer music tutorial. Cambridge: The MIT Press, 1999. Medio impreso.

Röbel, Axel. «Synthesizing Natural Sounds Using Dynamic Models of Sound Attractors». Computer Music Journal 25, 2 (2001). 46-61. Medio impreso.

Rowe, Robert. Machine Musicianship. Cambridge: The MIT Press, 2001. Medio impreso.

Singh, Nandini y Frederic Theunissen. "Modulation spectra of natural sounds and ethological theories of auditory processing». J Acoust Soc Am 114 (2003). 3.394-411. Medio impreso.

Schaeffer, Pierre. Traité des Objects Musicaux. París: Editions Du Seuil, 1966. Medio impreso.

Smith, Julius O. «Physical Modeling Using Digital Waveguides». Computer music journal 16, 4 (1992). 74-91. Medio impreso.

Stockhausen, Karlheinz. Stockhausen on music. Comp. Robin Maconie. Londres: Marion Boyars, 1989. Medio impreso.

Sturm, Bob. L. «Composing for an ensemble of atoms: the metamorphosis of scientific experiment into music». Organised Sound 6, 2 (2001). 131-45. Medio impreso.

Tanaka, Atau. «Musical Performance Practice on Sensor-based Instruments». Trends in Gestural Control of Music. Eds. Marcelo Wanderley y Marc Battier. París: IRCAM, 2000. Medio impreso.

Taube, Heinrich. Notes from the Metalevel. Introduction to Algorithmic Composition in Music. London: Taylor \& Francis, 2004. Medio impreso.

Turner, Tad. «The Resonance of the Cubicle: Laptop Performance in Post-digital Musics». Contemporary Music Review 22, 4 (2003). 81-92. Medio impreso.

Varèse, Edgar. "The Liberation of Sound». Audio Culture. Readings in Modern Music. Eds. Christoph Cox y Daniel Warner. Nueva York: Continuum, 2010. Medio impreso.

Weil, Benjamin. "Art in Digital Times: From Technology to Instrument». Leonardo 35, 2 (2002). 523-37. Medio impreso.

Winkler, Todd. Composing Interactive Music. Techniques and Ideas Using Max. Cambridge: The MIT Press, 1998. Medio impreso.

Wolek, Nathan. «A Simplified Interface for Granular Processing Based on Perceptual Research». Northwestern University, 2005. Tesis doctoral. 